Article

\title{
Cosmology with Varying Constants from a Thermodynamic Viewpoint
}

\author{
Hussain Gohar \\ Institute of Physics, University of Szczecin, Wielkopolska 15, 70-451 Szczecin, Poland; \\ Hussain.Gohar@usz.edu.pl \\ Academic Editors: Mariusz P. Dąbrowski, Manuel Krämer and Vincenzo Salzano \\ Received: 31 January 2017; Accepted: 13 March 2017; Published: 17 March 2017
}

\begin{abstract}
We study the variation of fundamental constants in cosmology while dealing with thermodynamic aspects of gravity. We focus on the variation of the speed of light, $c$, and Newton's gravitational constant, $G$, with respect to cosmic time. We find the constraints on the possible variation of these constants by comparing varying constants of cosmological models with the latest observational data.
\end{abstract}

Keywords: variation of fundamental constants; varying constants theories; gravity thermodynamics correspondence

\section{Introduction}

One of the most important breakthroughs in the field of black hole physics is the correspondence between the parameters of black holes and the quantities in thermodynamics. According to Hawking's area theorem, the area, $A$, of the surface of a black hole always increases so that we can relate it with the entropy, $S$, of the black hole by the relation $S=\left(k_{B} c^{3} / 4 G \hbar\right) A[1,2]$, where $k_{B}$ and $\hbar$ are Boltzmann's constant and reduced Planck constant, respectively. Classically, black holes do not emit radiation. By including the quantum effects, black holes do emit all kinds of radiation. This is why there is a temperature associated with the black hole, by the relation $T=\hbar \kappa / 2 \pi k_{B}$ [3], where $\kappa$ is the surface gravity of the black hole.

There are many approaches to studying the gravity-thermodynamic correspondence [4-8]. We focus on the approach given in $[9,10]$, in which the curvature of the space-time is proportional to the stress energy plus the entropic force terms (surface terms). The entropic terms are due to the force acting on the boundary of the universe due to the increasing entropy of the universe. However, this has been criticized on the basis of a galaxy formation problem and some other issues [11-14]. For extended versions of the entropic cosmology, see [15-17], where the authors consider the general framework of entropic cosmology, and these general entropic models solve the issues raised in [11-14].

The variation of fundamental constants has been a hot topic for the last twenty years. For a detailed review, see [18-20]. There are different approaches to studying the variation of fundamental constants in cosmology [21-29]. In this report, we briefly summarize the approach given by us in [29] to study the variation of fundamental constants. We extend the entropic cosmology to the variation of fundamental constants, the varying $c$ and varying $G$. We assume that the universe is a sphere with a boundary as Hubble horizon. We define the Hawking temperature and Bekenstein entropy on the boundary. We further assume that the Hawking temperature and Bekenstein entropy additionally depend on varying $c$ and varying $G$. We use the first law of thermodynamics to find the continuity equation, and use it to find the entropic force terms in the field equations by following the papers [15-17]. We compare our model with the data from supernovae, Baryon Acoustic Oscillations (BAO), and Cosmic Microwave Background (CMB). Our model is also related to dynamical vacuum energy models, which have been 
discussed and confronted with data [11-14,30-32]. Similarly, dynamical dark energy models with varying fundamental constants have been discussed in $[33,34]$. The remainder of the report is as follows. In Section 2, we give the modified field equations for varying constants theories from a thermodynamic point of view. In Section 3, we compare our model with the latest observational data and find the bounds on varying $c$ and $G$, and in Section 4 , we give the conclusion.

\section{Modified Field Equations}

We consider the universe as a sphere of volume $V$ with a boundary or screen as Hubble horizon. We associate the temperature and entropy with the boundary of the universe. We apply the Hawking temperature $T$ and the Bekenstein entropy $S$ on the boundary, which additionally depend on varying $c$ and $G$ with respect to cosmic time

$$
T=\frac{\gamma \hbar c(t)}{2 \pi k_{B} r_{h}(t)}, \quad S=\frac{k_{B}}{4 \hbar}\left(\frac{c^{3}(t) A(t)}{G(t)}\right) .
$$

Here $A(t)=4 \pi r_{h}^{2}(t)$, where $r_{h}(t) \equiv c(t) / H(t)$ is the Hubble horizon, $H(t)$ is the Hubble parameter, and $\gamma$ is a non-negative parameter on the order of unity $O(1)$ associated with the boundary of the universe. By using the first law of thermodynamics for non adiabatic expansion of the universe

$$
\frac{d E}{d t}+p \frac{d V}{d t}=T \frac{d S}{d t}
$$

we have the first modified continuity equation

$$
\dot{\rho}+3 H\left(\rho+\frac{p}{c^{2}(t)}\right)=-2 \frac{\dot{c}(t)}{c(t)} \rho+\frac{3 \gamma H^{2}}{8 \pi G(t)}\left(5 \frac{\dot{c}(t)}{c(t)}-\frac{\dot{G}(t)}{G(t)}-2 \frac{\dot{H}}{H}\right)
$$

$d V / d t=3 V(t) H(t)$ and the internal energy $E(t)=\varepsilon(t) V(t)$, where energy density $\varepsilon(t)=\rho(t) c^{2}(t)$, are being used to get the above modified continuity Equation (3), and $a$ is the scale factor. We follow the formulation of entropic cosmology ([15-17] and references therein) to find the entropic force terms. For detailed calculations, see [29]. Let us assume that the general functions $f(t)$ and $g(t)$ are the additional terms in Friedmann and acceleration equations

$$
\begin{aligned}
& \left(\frac{\dot{a}}{a}\right)^{2}=\frac{8 \pi G(t)}{3} \rho-\frac{k c^{2}(t)}{a^{2}}+f(t), \\
& \frac{\ddot{a}}{a}=-\frac{4 \pi G(t)}{3}\left(\rho+\frac{3 p}{c^{2}(t)}\right)+g(t) .
\end{aligned}
$$

The functions $f(t)$ and $g(t)$ play a role analogous to bulk viscosity. In our framework, these functions play the role of entropic force terms and the terms due to the variation of $c$ and $G$. From Friedmann Equation (4) and acceleration Equation (5), we obtain the second modified continuity equation

$$
\dot{\rho}+3 H\left(\rho+\frac{p}{c^{2}(t)}\right)+\rho \frac{\dot{G(t)}}{G(t)}-3 \frac{k c(t) \dot{c}(t)}{4 \pi G(t) a^{2}(t)}=\frac{3 H}{4 \pi G(t)}\left(g(t)-f(t)-\frac{\dot{f}(t)}{2 H}\right) .
$$

We can see that generalized continuity Equation (6) has dissipative terms similar to bulk viscosity models. We define the functions

$$
f(t)=\gamma H^{2} \text { and } g(t)=\gamma H^{2}+\frac{\gamma}{2}\left(5 \frac{\dot{c}(t)}{c(t)}-\frac{\dot{G}(t)}{G(t)}\right) H+\frac{4 \pi G(t)}{3 H}\left(\frac{\dot{G}(t)}{G(t)}-2 \frac{\dot{c}(t)}{c(t)}\right) \rho
$$


by comparing the Equations (3) and (6), so we have the modified Friedmann Equation (4) and acceleration Equation (5),

$$
\begin{gathered}
\left(\frac{\dot{a}}{a}\right)^{2}=\frac{8 \pi G(t)}{3} \rho+\gamma H^{2}, \\
\frac{\ddot{a}}{a}=-\frac{4 \pi G(t)}{3}\left(\rho+\frac{3 p}{c^{2}(t)}\right)+\gamma H^{2}+\left(\frac{7 \gamma-2}{2}\right) \frac{\dot{c}(t)}{c(t)} H+\left(\frac{1-2 \gamma}{2}\right) \frac{\dot{G}(t)}{G(t)} H,
\end{gathered}
$$

together with modified continuity equation

$$
\dot{\rho}+3 H\left(\rho+\frac{p}{c^{2}(t)}\right)=-2 \frac{\dot{c}(t)}{c(t)} \rho+\frac{3 \gamma H^{2}}{8 \pi G(t)}\left(5 \frac{\dot{c}(t)}{c(t)}-\frac{\dot{G}(t)}{G(t)}-2 \frac{\dot{H}}{H}\right) .
$$

If we use the ansätze $c=c_{0} a^{n}$ and $G=G_{0} a^{q}$ in the above-modified equations of motion, then these equations are analogous to the entropic force expressions [15-17] and running vacuum models [11-14,30-32]. We consider the multiple fluid scenario, and using the barotropic equation of state $p_{i}=w_{i} \rho_{i}$, we solve the modified continuity equation

$$
\rho_{i}=\rho_{0}\left(\frac{H}{H_{0}}\right)^{\frac{2 \gamma}{1-\gamma}} a_{i} f_{i}^{X}(\gamma, n, q),
$$

where $f_{i}^{c}(\gamma, n)=-3\left(1+w_{i}+\frac{n(2-7 \gamma)}{3(1-\gamma)}\right)$ is for varying $c$, and $f_{i}^{G}(\gamma, q)=-3\left(1+w_{i}+\frac{q \gamma}{3(1-\gamma)}\right)$ is for varying $G$. Here $n, q$, and $\gamma$ are free parameters which can be constrained from the observations. For each fluid with the barotropic parameter $w_{i}$ and barotropic equation of state $\rho_{i}=w_{i} \rho_{i}$, we have $w_{i}=0$ for matter, $w_{i}=1 / 3$ for radiation, and $w_{i}=-1$ for vacuum. Now, the normalized Friedmann equations can be written as

$$
E^{2}=\left(\frac{H}{H_{0}}\right)^{2}=\left(\sum_{i} \frac{\Omega_{i, 0}}{1-\gamma} a_{i}^{c}(\gamma, n)\right)^{\frac{\gamma-1}{2 \gamma-1}}
$$

for varying $c$ model and

$$
E^{2}=\left(\frac{H}{H_{0}}\right)^{2}=\left(a^{q} \sum_{i} \frac{\Omega_{i, 0}}{1-\gamma} a_{i}^{G}(\gamma, q)\right)^{\frac{\gamma-1}{2 \gamma-1}}
$$

for varying $G$ model, where $\Omega_{i, 0}=\left(8 \pi \rho_{i, 0} / 3 H_{0}^{2}\right)$.

\section{Observational Data Analysis}

The analysis has involved the largest updated set of cosmological data available, and includes: Type Ia Supernovae (SNeIa); Baryon Acoustic Oscillations (BAO); Cosmic Microwave Background (CMB); and a prior on the Hubble constant parameter, $H_{0}$. For detailed calculations and analysis, see [29]. The main results of observational analysis are given in Table 1. We have found the observational bound on the free parameter $\gamma$, which is taken to the order of unity on the theoretical background. Our analysis shows that it should be on the order of $10^{-2}-10^{-4}$, and this is consistent with the results obtained in [31]. Similarly, we have found the bound on the variability of $c$ and G. Our analysis shows that both $c$ and $G$ are increasing with the evolution of the universe in the entropic description. 
Table 1. Observational parameters of the models under study.

\begin{tabular}{cccccccccc}
\hline id. & $\mathbf{\Omega}_{m}$ & $\boldsymbol{\Omega}_{\mathbf{b}}$ & $\mathbf{h}$ & $\mathbf{q} / \mathbf{n}$ & $\gamma$ & $\alpha$ & $\beta$ & $\mathcal{M}_{\mathbf{B}}^{\mathbf{1}}$ & $\boldsymbol{\Delta}_{\mathbf{m}}$ \\
\hline$G=G_{0} a^{q}$ & $0.314_{-0.008}^{+0.009}$ & $0.0453_{-0.0009}^{+0.0009}$ & $0.698_{-0.007}^{+0.007}$ & $0.048_{-0.033}^{+0.042}$ & $<0.022$ & $0.141_{-0.006}^{+0.007}$ & $3.106_{-0.087}^{+0.077}$ & $-19.044_{-0.019}^{+0.018}$ & $-0.071_{-0.023}^{+0.023}$ \\
$c=c_{0} a^{n}$ & $0.311_{-0.007}^{+0.007}$ & $0.046_{-0.001}^{+0.001}$ & $0.696_{-0.007}^{+0.007}$ & $0.00049_{-0.00053}^{+0.00049}$ & $<0.0007$ & $0.141_{-0.007}^{+0.007}$ & $3.100_{-0.080}^{+0.080}$ & $-19.043_{-0.018}^{+0.018}$ & $-0.070_{-0.022}^{+0.023}$ \\
\hline
\end{tabular}

Recently, it has been claimed that the fine structure constant $\alpha=e^{2} / \hbar c$ (do not confuse this $\alpha$ with the $\alpha$ in Table $1-\alpha$ and $\beta$ in Table 1 characterize the stretch luminosity and color luminosity relationship) is changing [35-39] (where $e$ is the electron charge), so relating the speed of light with the fine structure constant, we have

$$
\frac{\Delta c}{c}=-\frac{\Delta \alpha}{\alpha}=n \frac{\Delta a}{a} \sim \frac{n}{10} .
$$

From Table 1, we evaluate $n=4.9 \times 10^{-4}>0$, and this implies that a change in $c$ (or $\alpha$ ) from our fit is $\Delta \alpha / \alpha_{0} \sim 10^{-5}$ in the redshift range [1;2], while other observational bounds in the same range (see Table II of [40] and [36-39]) give $\Delta \alpha / \alpha_{0} \sim 10^{-6}$. However, our estimation is compatible with constraints from CMB Planck first release [41]. Similarly, for $G$, we have $q=0.048$ from Table 1 , and this can be written as $(\dot{G} / G)_{0}=q H_{0} \sim 3.42 \times 10^{-12}$ year $^{-1}$ by using the current value of the Hubble constant $H_{0}$. This is compatible with the Solar System bound coming from Viking landers on Mars [42] $(\dot{G} / G)_{0}=(2 \pm 4) \times 10^{-12}$ year $^{-1}$ and weaker than the bound from the Lunar laser ranging $[43](\dot{G} / G)_{0}=(4 \pm 9) \times 10^{-13}$ year $^{-1}$.

Recently, new methods [44-46] have been introduced to detect the possible variation of speed of light by using future galaxy surveys, such as The Square Kilometer Array (SKA), Euclid, and the Wide-Field Infrared Survey Telescope (WFIRST-2.4). For example, in [45] it is shown that SKA will be able to detect a $1 \%$ variation in the speed of light at $3 \sigma$ level.

\section{Conclusions}

In this report, we discussed entropic cosmology with varying gravitational constant $G$ and varying speed of light $c$. We discussed the consequences of these variations on the entropic force terms. We started with a general set of field equations which described varying constants' entropic cosmology. We derived the continuity equation from the first law of thermodynamics by using the Bekenstein entropy, as well as Hawking temperature to fit the general entropic terms to this continuity equation. We have examined many-fluid entropic models against observational data (supernovae, BAO, and CMB). We have used data from JLA compilation of SDSS-II and SNLS collaboration (supernovae), WiggleZ Dark Energy Survey, and SDSS-III Baryon Oscillation Spectroscopic Survey (BOSS), as well as Planck data (CMB). We found that the observational bound on the Hawking temperature coefficient $\gamma$ was much smaller $\left(10^{-2}-10^{-4}\right)$ than it is usually assumed to be on the theoretical basis to be on the order of unity $O(1)$. We have also found that in our entropic models, $G$ and $c$ should be increasing with the evolution of the universe. Our bound on the variation of $c$ being $\Delta c / c \sim 10^{-5}$ is at least one order of magnitude weaker than observational bound obtained from analysis of the quasar spectra.

Acknowledgments: This project was financed by the Polish National Science Center (NCN) Grant DEC-2012/06/A/ST2/00395 and NCN doctoral scholarship (Agreement number: UMO-2016/20/T/ST2/00490) granted within the framework of ETIUDA 4.

Conflicts of Interest: The author declares no conflict of interest.

\section{References}

1. Bekenstein, J.D. Black holes and entropy. Phys. Rev. D 1973, 7, 2333-2346.

2. Bekenstein, J.D. Generalized second law of thermodynamics in black hole physics. Phys. Rev. D 1974, 9, 3292-3300.

3. Hawking, S.W. Black hole explosions. Nature 1974, 248, 30-31. 
4. Jacobson, T. Thermodynamics of space-time: The Einstein equation of state. Phys. Rev. Lett. 1995, 75, 1260-1263.

5. Padmanabhan, T. Gravitational entropy of static space-times and microscopic density of states. Class. Quantum Gravity 2004, 21, 4485-4494.

6. Padmanabhan, T. Thermodynamical Aspects of Gravity: New Insights. Rep. Prog. Phys. 2010, 73, 046901.

7. Verlinde, E.J. On the Origin of Gravity and the Laws of Newton. J. High Energy Phys. 2011, 2011, 1-27.

8. Verlinde, E.J. Emergent Gravity and the Dark Universe. arXiv 2016, arXiv:1611.02269.

9. Easson, D.A.; Frampton, P.H.; Smoot, G.F. Entropic Accelerating Universe. Phys. Lett. B 2011, 696, $273-277$.

10. Easson, D.A.; Frampton, P.H.; Smoot, G.F. Entropic Inflation. Int. J. Mod. Phys. A 2012, 27, 125066.

11. Basilakos, S.; Polarski, D.; Solá, J. Generalizing the running vacuum energy model and comparing with the entropic-force models. Phys. Rev. D 2012, 86, 043010.

12. Basilakos, S.; Solá, J. Entropic-force dark energy reconsidered. Phys. Rev. D 2014, 90, 023008.

13. Gómez-Valent, A.; Solá, J. Vacuum models with a linear and a quadratic term in H: Structure formation and number counts analysis. Mon. Not. R. Astron. Soc. 2015, 448, 2810-2821.

14. Basilakos, S.; Plionis, M.; Solá, J. Hubble expansion and Structure Formation in Time Varying Vacuum Models. Phys. Rev. D 2009, 80, 083511.

15. Komatsu, N.; Kimura, S. Non-adiabatic-like accelerated expansion of the late universe in entropic cosmology. Phys. Rev. D 2013, 87, 043531.

16. Komatsu, N.; Kimura, S. Entropic cosmology for a generalized black-hole entropy. Phys. Rev. D 2014, $88,083534$.

17. Komatsu, N.; Kimura, S. Evolution of the universe in entropic cosmologies via different formulations. Phys. Rev. D 2014, 89, 123501.

18. Uzan, J.-P. Varying constants, gravitation, and cosmology. Liv. Rev. Rel. 2011, 14, 2.

19. Ellis, G.F.R.; Uzan, J.-P. $c$ is the speed of light, isn't it? Am. J. Phys. 2005, 73, 240-247.

20. Duff, M.J. How fundamental are fundamental constants? J. Contemp. Phys. 2015, 56, 35-47.

21. Albrecht, A.; Magueijo, J. A Time varying speed of light as a solution to cosmological puzzles. Phys. Rev. D 1999, 59, 043516.

22. Barrow, J.D.; Magueijo, J. Solutions to the quasi-flatness and quasilambda problems. Phys. Lett. B 1999, 447, 246-250.

23. Barrow, J.D. Cosmologies with varying light speed. Phys. Rev. D 1999, 59, 043515.

24. Moffat, J. Superluminary universe: A possible solution to the initial value problem in cosmology. Int. J. Mod. Phys. D 1993, 2, 351-366.

25. Clayton, M.A.; Moffat, J.W. Fluctuation Spectrum from a Scalar-Tensor Bimetric Gravity Theory. Int. J. Mod. Phys. D 2003, 12, 697-712.

26. Moffat, J.W. Bimetric Gravity Theory, Varying Speed of Light and the Dimming of Supernovae. Int. J. Mod. Phys. D 2003, 12, 281-298.

27. Hossenfelder, S. Bounds on an energy-dependent and observer-independent speed of light from violations of locality. Phys. Rev. Lett. 2010, 104, 140402.

28. Magueijo, J.; Smolin, L. Gravity's rainbow. Class. Quantum Gravity 2004, 21, 1725-1736.

29. Dąbrowski, M.P.; Gohar, H.; Salzano, V. Varying Constants Entropic- $\Lambda$ CDM Cosmology. Entropy 2016, 18, 60.

30. Grande, J.; Solá, J.; Basilakos, S.; Plionis, M. Hubble expansion and structure formation in the "running FLRW model" of the cosmic evolution. J. Cosmol. Astrop. Phys. 2011, 2001, 007.

31. Gómez-Valent, A.; Solá, J.; Basilakos, S. Dynamical vacuum energy in the expanding Universe confronted with observations: A dedicated study. J. Cosmol. Astrop. Phys. 2015, 2015, 004.

32. Solá, J.; Gómez-Valent, A.; de Cruz Pérez, J. Hints of dynamical vacuum energy in the expanding Universe. Astrophys. J. Lett. 2015, 811, L14.

33. Fritzsch, H.; Solá, J.; Nunes, R.C. Running vacuum in the Universe and the time variation of the fundamental constants of Nature. arXiv 2016, arXiv:1605.06104.

34. Grande, J.; Solá, J.; Fabris, J.C.; Shapiro, I.L. Cosmic perturbations with running $G$ and $\Lambda$. Class. Quantum Gravity 2010, 27, 105004.

35. Webb, J.K.; King, J.A.; Murphy, M.T.; Flambaum, V.V.; Carswell, R.F.; Bainbridge, M.B. Indications of a spatial variation of the fine structure constant. Phys. Rev. Lett. 2011, 107, 191101 
36. Molaro, P.; Centurion, M.; Whitmore, J.B.; Evans, T.M.; Murphy, M.T.; Agafonova, I.I.; Bonifacio, P.; D'Odorico, S.; Levshakov, S.A.; Lopez, S.; et al. The UVES Large Program for Testing Fundamental Physics: I Bounds on a change in $\alpha$ towards quasar HE 2217-2818. Astron. Astrophys. 2013, 555, A68.

37. Molaro, P.; Reimers, D.; Agafonova, I.I.; Levshakov, S.A. Bounds on the fine structure constant variability from Fe II absorption lines in QSO spectra. Eur. Phys. J. Spec. Top. 2008, 163, 173-189.

38. Chand, H.; Srianad, R.; Petitjean, P.; Aracil, B.; Quast, R.; Reimers, D. On the variation of the fine-structure constant: Very high resolution spectrum of QSO HE 0515-4414. Astron. Astrophys. 2006, 451, 45-56.

39. Agafonova, I.I.; Molaro, P.; Levshakov, S.A. First measurement of Mg isotope abundances at high redshifts and accurate estimate of $\Delta \alpha / \alpha$. Astron. Astrophys. 2011, 529, A28.

40. Dasbrowski, M.P.; Denkiewicz, T.; Martins, C.J.A.P.; Vielzeuf, P. Variations of the fine-structure constant $\alpha$ in exotic singularity models. Phys. Rev. D 2014, 89, 123512.

41. O'Brian, J.; Smidt, J.; de Bernardis, F.; Cooray, A. Constraints on Spatial Variations in the Fine-Structure constant from Planck. Astrophys. J. 2015, 798, 18.

42. Hellings, R.W.; Adams, P.J.; Anderson, J.D.; Keesey, M.S.; Lau, E.L.; Standish, E.M.; Canuto, V.M.; Goldman, I. Experimental Test of the Variability of G Using Viking Lander Ranging Data. Phys. Rev. Lett. 1983, 51, 1609.

43. Williams, J.G.; Turyshev, S.G.; Boggs, D.H. Progress in lunar laser ranging tests of relativistic gravity. Phys. Rev. Lett. 2004, 93, 26101.

44. Salzano, V.; Dąbrowski, M.P.; Lazkoz, R. Measuring the speed of light with Baryon Acoustic Oscillations. Phys. Rev. Lett. 2015, 114, 101304.

45. Salzano, V.; Dąbrowski, M.P.; Lazkoz, R. Probing the constancy of the speed of light with future galaxy survey: The case of SKA and Euclid. Phys. Rev. D 2016, 93, 063521.

46. Dąbrowski, M.P.; Salzano, V.; Balcerzak, A.; Lazkoz, R. New tests of variability of the speed of light. EPJ Web Conf. 2016, 126, 04012.

(C) 2017 by the authors. Licensee MDPI, Basel, Switzerland. This article is an open access article distributed under the terms and conditions of the Creative Commons Attribution (CC BY) license (http:/ / creativecommons.org/licenses/by/4.0/). 